\title{
Non-perturbative string connections
}

\author{
Andrea Gregori \\ Università di Milano-Bicocca \\ via Celoria 16, 20133 Milano
}

ABSTRACT: We discuss the duality between two type I compactifications to four dimensions and an heterotic construction with spontaneous breaking of the $\mathcal{N}=4$ supersymmetry to $\mathcal{N}=2$. This duality allows us to gain insight into the non-perturbative properties of these models. Through the analysis of the gravitational corrections, we then investigate the connections between four-dimensional, $N=2$ M-theory vacua constructed as orbifolds of type II, heterotic, and type I strings.

After the "second string revolution", that took place after 1995 , it is a common belief that all the string constructions are manifestations of a unique underlying theory. In most of the cases, the different string models correspond to different regions in the moduli space of the underlying theory. There are however several cases in which apparently disconnected vacua turn out to be indeed equivalent, being simply related by a "change of parametrization".

In some cases, this reparametrization maps perturbative moduli into non-perturbative ones. In these cases, knowing this map allows to compute in an easy way quantities that would be beyond a perturbative, often short, computation. This is the case for instance of the duality between the heterotic string compactified to four dimensions and the type IIA string compactified on a K3 fibration [1]. The heterotic dilatonaxion field is mapped into a perturbative modulus, associated to the volume form of the base of the fibration, on the type IIA side. Therefore, what is non-perturbative on the heterotic side is perturbative on the type IIA side. This relation, that recently received a further confirmation [i2], has been used in order to compute the non-perturbative correction to the effective coupling of the $R^{2}$ term in some specific examples [3]- $-[\overline{6}$ i $]$ (An analogous relation exists also between some type II asymmetric orbifold compactifications and the type IIA string $[\overline{6}|-\overline{6}| \overline{0} \mid]$. However, the cases in which such duality exists are far from covering the main part of the heterotic constructions. It seems indeed that some of the most interesting cases don't fall in this class. The question is therefore whether it is nevertheless possible, in some of these cases, to obtain (at least partial) information on the nonperturbative behavior. We present here an analysis of a four-dimensional, $\mathcal{N}=2$ heterotic construction which, although without type IIA dual, possesses nevertheless a pair of type I string duals. Putting together the informations coming from all these constructions, it is possible to obtain partial but not irrelevant informations about the non-perturbative behavior of this model.

Through the analysis of the gravitational corrections, we discuss then the connections of these constructions with other type II and heterotic constructions.

The heterotic model we consider is constructed as a $Z_{2}$, "freely acting" orbifold of the string compactified on $T^{6}=T^{2} \times T^{4}$. The $Z_{2}$ projection acts as a reflection, $x_{i} \rightarrow-x_{i}$, on $T^{4}$ and as a half-circumference translation in one circle of $T^{2}$. Imposing modular invariance and requiring the shift of the momenta of the $T^{2}$-lattice produced by this translation to be left-right symmetric, leads to a specific embedding of the spin connection into the gauge group, such that, at the orbifold point, the massless spectrum origi- 
nating from the $c=(0,16)$ currents always contains an equal number of vector and hypermultiplets. This equality is however not due to a "level two" realization of the gauge group: the rank is sixteen, i.e. the maximal allowed in perturbative heterotic constructions. The free action of $Z_{2}$ produces then a spontaneous breaking of the $\mathcal{N}=4$ supersymmetry, that can be restored when the translated coordinated is decompactified. This corresponds to a special limit in the space of the moduli of the two-torus, $T$ and $U$, associated respectively to the Kähler class and the complex structure. At the $U(1)^{16}$ point, this model has sixteen vector multiplets and sixteen hypermultiplets from the currents, plus the three vectors and four hypermultiplets from the compact space. Due to the free action of $Z_{2}$, there are no massless states originating from the twisted sector. Although it exists a type IIA orbifold construction with the same massless spectrum, obtained by compactification on an orbifold limit of a Calabi Yau manifold with Hodge numbers $(19,19)$, this is not dual to the heterotic orbifold; the CY ${ }^{19,19}$ manifold is not in fact a K3 fibration.

The heterotic orbifold possesses on the other hand a type I dual, constructed as an orientifold of the $\mathcal{N}=4$, type IIB string, in which the $\mathcal{N}=8$ supersymmetry is spontaneously broken by a $Z_{2}$ freely acting projection, whose action of $T^{6}$ is the same as that of the heterotic model. In the case of the heterotic string, T-duality makes irrelevant the choice of the translation, that can equivalently be performed on the momenta, projection $(-1)^{m}$, or on the windings, projection $(-1)^{n}$; in the case of type I orientifolds on the other hand the two projections lead to rather different models $[\overline{9}]$. In the first case, the model obtained is exactly equivalent to the heterotic one, with a perturbative, spontaneous breaking of the $\mathcal{N}=4 \mathrm{su}-$ persymmetry and a gauge group of rank sixteen, originating entirely from D9-branes: there are no D5-branes. By introducing appropriate Wilson lines it is possible to obtain exactly the same spectrum of the heterotic construction. The type I orientifold obtained from the type IIB string with translation $(-1)^{n}$, on the other hand, has still a gauge group of rank sixteen, but that now originates half from the D9-branes sector and half from the D5-branes sector. Moreover, the spec- trum on the branes doesn't feel the breaking of supersymmetry. This model doesn't look therefore dual to the previous constructions.

In order to investigate the conjectured duality between the heterotic and the first of the two type I constructions, we consider the string corrections to the effective coupling of the $R^{2}$ term. As it was discussed in Refs. $[\overline{1}-1,160$ order to compare string constructions, it is necessary to project out the non-universal contribution coming from the coupling of the "bulk" sector with the currents. This can be consistently done because the contribution of the "currents" to the gravitational and gauge couplings are proportional, and can be subtracted by properly redefining the amplitude we compute. On the heterotic side, a further contribution must be subtracted, namely that coming at the singularities in the space of the moduli $T$ and $U$ : at these points, new vector and/or hypermultiplets appear. This phenomenon doesn't happen on the type I side. Once this projection has been performed, the renormalization of the gravitational amplitudes read, in the two models:

Het. :

$$
\begin{aligned}
\frac{16 \pi^{2}}{g^{2}}= & 16 \pi^{2} \Im S-2 \log \Im T\left|\vartheta_{4}(T)\right|^{4} \\
& -2 \log \Im U\left|\vartheta_{4}(U)\right|^{4} \\
& +\mathcal{O}(\log \mu / M)
\end{aligned}
$$

Type I :

$$
\begin{aligned}
\frac{16 \pi^{2}}{g^{2}}= & 16 \pi^{2} \Im S-2 \log \Im U\left|\vartheta_{4}(U)\right|^{4} \\
& +\mathcal{O}(\log \mu / M) .
\end{aligned}
$$

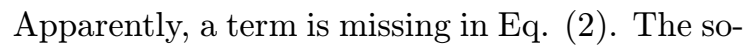
lution to this puzzle comes from the observation that the second term in the r.h.s. of Eq.(高京) behaves, for large- $T$, as: $\log \Im T\left|\vartheta_{4}(T)\right|^{4} \sim \log \Im T$. There is no linear divergence in $T$, and the logarithmic behavior can be interpreted as due to non-perturbative phenomena. Indeed, it can be removed by switching on an appropriate infrared

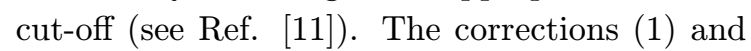
(12) are therefore consistent with the duality of these constructions. We may however ask what is indeed the meaning of the heterotic field $T$, whose contribution is apparently lost when go- 
ing to the type I dual. In the opposite limit, $T \rightarrow 0$, the heterotic correction $\left(\begin{array}{l}\overline{1} \\ 1 \\ 1\end{array}\right)$ diverges linearly in the inverse field, $\tilde{T} \equiv-1 / T$. By redefining $\tilde{T} \equiv S^{\prime}$, we see that in the large $\tilde{T}$ limit, $\left(\underline{1}^{\prime}{ }^{\prime}\right)$ behaves as:

$$
\begin{aligned}
\frac{16 \pi^{2}}{g^{2}} \approx & 16 \pi^{2} \Im S+16 \pi^{2} \Im S^{\prime} \\
& -2 \log \Im U\left|\vartheta_{4}(U)\right|^{4} .
\end{aligned}
$$

This is indeed the behavior of a type I model with both D9- and D5-branes sectors. Actually, this is the correction as it would be computed on the second type I construction. We guess that indeed the second type I construction is dual to the heterotic and the other type I model. This hypothesis is supported by a look at the path followed by the D5-branes when their coupling, parametrized by $S^{\prime}$, becomes very weak $\left(S^{\prime} \rightarrow 0\right)$ [100"]. The crucial point is that this model can be viewed as obtained via a freely acting $Z_{2}$ projection performed along the eleventh coordinate of the M-theory [9.9]. In this model, owing to the free action of this projection, $\mathrm{S}\left(\mathrm{S}^{\prime}\right)$-duality is broken. Indeed, in the limit $S^{\prime} \rightarrow 0$, that corresponds to a decompactification of the $T^{4}$, the D5-branes massless fields are still present in the spectrum. However, being the D5-branes coupling missing, these states have to be interpreted as originating from D9-branes. Supersymmetry of the branes spectrum is indeed an artifact, due to the separation into closed and open sectors, typical of type I constructions. It seems therefore possible to imagine to break supersymmetry only on the bulk, represented by the closed string sector. However, through interactions of the bulk fields with the fields living on the branes, the breaking of supersymmetry is then communicated to the whole theory. It is indeed from the heterotic dual, where there is no such a fake separation, that we learn how the supersymmetry breaking is actually communicated from one sector to the other.

The relation to the M-theory is better understood by going to the dual, type $\mathrm{I}^{\prime}$ picture, where D9- and D5-branes appear as D4- and D8branes, and the eleventh coordinate corresponds to the tenth coordinate of the string. From the M-theory point of view, the freely acting, ScherkSchwarz supersymmetry breaking projection on the eleventh coordinate leaves untouched, at least in a first approximation, the two "Hořava-Witten walls", whose massless fields correspond respectively to the D4- and D8-branes fields [īi]. Since the system is symmetric under reflection of the eleventh coordinate, we argue that the breaking of the $\mathrm{S}^{\prime}$-duality on the D4 sector has a mirror in an analogous breaking of S-duality on the D8branes sector. Under this hypothesis, the correction given in Eq. (1יiln ) should be promoted to:

$$
\begin{aligned}
\frac{16 \pi^{2}}{g^{2}}= & -2 \log \Im \tilde{S}\left|\vartheta_{4}(\tilde{S})\right|^{4}-2 \log \Im \tilde{S}^{\prime}\left|\vartheta_{4}\left(\tilde{S}^{\prime}\right)\right|^{4} \\
& -2 \log \Im U\left|\vartheta_{4}(U)\right|^{4}+\mathcal{O}\left(\mathrm{e}^{-\left(\tilde{S}, \tilde{S}^{\prime}, U\right)}\right),
\end{aligned}
$$

where $\tilde{S} \equiv-1 / S, \tilde{S}^{\prime} \equiv-1 / S^{\prime}$, and the last term is a series of exponentials, symmetric in the three fields, suppressed in the large and small fields limits. This is the typical behavior of a theory with spontaneous breaking of the $\mathcal{N}=8$ supersymmetry. Expression ( $\left(\begin{array}{l}1 \\ 1\end{array}\right)$ diverges linearly for large- $S$, large- $S^{\prime}$ (and small $U$ ), but only logarithmically for large $\tilde{S}, S^{\prime}, U$. This is the limit of restoration of the $\mathcal{N}=8$ supersymmetry.

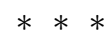

The analogous gravitational correction in the type IIA orbifold with Hodge numbers $(19,19)$, is

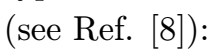

$$
\begin{aligned}
\frac{16 \pi^{2}}{g^{2}}= & -2 \log \Im T^{(1)}\left|\vartheta_{4}\left(T^{(1)}\right)\right|^{4} \\
& -6 \log \Im T^{(2)}\left|\eta\left(T^{(2)}\right)\right|^{4} \\
& -6 \log \Im T^{(3)}\left|\eta\left(T^{(3)}\right)\right|^{4},
\end{aligned}
$$

where $T^{(1)}, T^{(2)}, T^{(3)}$ are the moduli associated to the Kähler classes of the three tori of $T^{6}=$ $T^{2} \times T^{2} \times T^{2}$, and we omit for simplicity the term logarithmically dependent on the infrared cut-off. It is clear that this correction doesn't match the heterotic one. Indeed, this type IIA orbifold is dual to a type II asymmetric orbifold, in which the modulus $T^{(1)}$ plays the role of the dilatonaxion field. It is also clear that, if the heterotic model has a spontaneously broken $\mathcal{N}=8$ supersymmetry, it cannot possess a type IIA dual, in which this super-Higgs phenomenon would appear as perturbative. Indeed, a type IIA orbifold 
with the spontaneous breaking of $\mathcal{N}=8$ exists: it is a $Z_{2} \times Z_{2}$ orbifold in which both the projec-

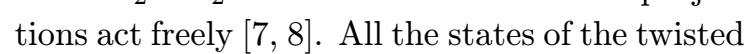
sectors are massive, and the massless spectrum contains only, besides the gravity multiplet, three vector multiplets and four hypermultiplets. The gravitational correction reads:

$$
\begin{aligned}
\frac{16 \pi^{2}}{g^{2}}= & -2 \log \Im T^{(1)}\left|\vartheta_{4}\left(T^{(1)}\right)\right|^{4} \\
& -2 \log \Im T^{(2)}\left|\vartheta_{4}\left(T^{(2)}\right)\right|^{4} \\
& -2 \log \Im T^{(3)}\left|\vartheta_{4}\left(T^{(3)}\right)\right|^{4}
\end{aligned}
$$

\section{Acknowledgments}

This talk is based on a work done in collaboration with C. Kounnas; I take this opportunity to address my thanks to him, and to acknowledge the EEC, under the contract TMR-ERBFMRXCT96-0045, the Swiss National Science Foundation and the Swiss Office for Education and Science, for financial support.

\section{References}

[1] C.M. Hull and P.K. Townsend, 'Nucl. Phys. B',

In a particular limit in the moduli space $\left(\bar{T}^{11)} \rightarrow\right.$ 0), this orbifold behaves as a K3 fibration, and

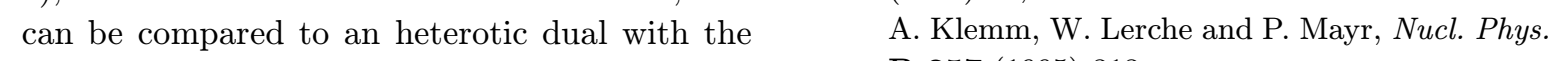

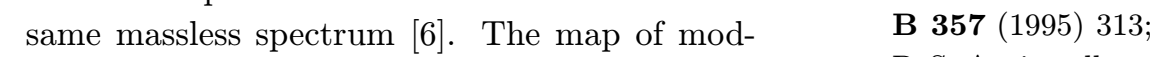

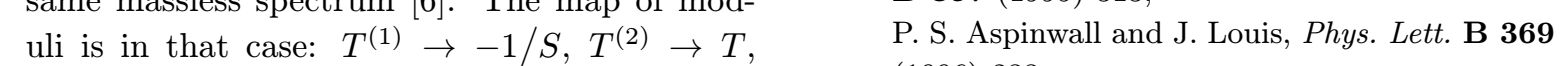

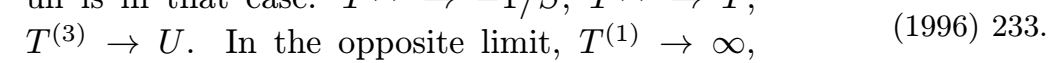

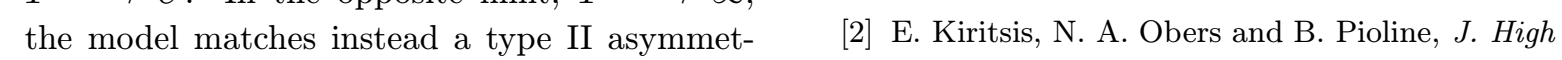
- ric_freely_acting_orbifold,_with_map_of_moduli __ $\underline{T}_{-}^{(1)} \rightarrow-S, T_{-}^{(2)} \rightarrow T_{2} \underline{T}_{-}^{(3)} \rightarrow U_{-}$Heterotic and

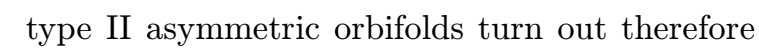
to be S-dual the one to the other.

Although not coinciding in the "bulk" of the

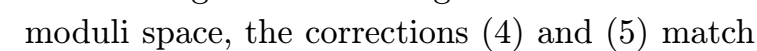
at the corner of moduli space, precisely at the limit in which the supersymmetry breaking $Z_{2}$ projection of the heterotic/type I side ends to act freely: in this limit, corresponding to $S^{\prime} \rightarrow \infty$,

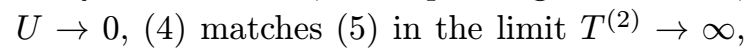
$T^{(3)} \rightarrow \infty$. This is however not evidence that at the limit the two theories really match: indeed, when we recover a genuine orbifold limit, we expect new massless states to appear, associated to the orbifold fixed points ${ }^{1}$. On the other _ band, _it_is _possible to interpolate_ between _the ' freely-acting- type- ILA/heterotic/type _II-asymmetric orbifolds and the heterotic/type I I con'structions_with_rank_16_ by switching_on an_appropriate Wilson line that, already at the $\mathcal{N}=4$ level, lifts the mass of all the fields originating from the currents. It turns out therefore that : some of these constructions can be connected by passing through appropriate limits in the moduli spaces.

\footnotetext{
${ }^{1}$ In the light of recent investigations, this part of the conclusions presented in [10] needs a slight modification.
}

Energy Phys. 0001 (2000) 029!

[3] J. A. Harvey and G. Moore, Phys. Rev. D. 57' (1998) 2323'; 'Phys. Rev. D_57_(1998)_23291.

[4] A. Gregori, E. Kiritsis, C. Kounnas, N.A. Obers, P.M. Petropoulos and B. Pioline, 'Nucl. Phys. Bi 510 (1998) 423.

[5] A. Gregori, C. Kounnas and P.M. Petropoulos, Nucl. Phys. B $\mathbf{5} \overline{\mathbf{3}} \overline{\mathbf{7}}(\overline{1999)} \overline{3} \overline{1}$;

A. Gregori, Talk given at 6th Hellenic School and Workshop on Elementary Particle Physics:, Corfu, Greece, 6-26 Sep 1998. 3In Corfu 1998, Quantum aspects of gauge theories, supersymmetry and unification, 348-355, hep-th/9811096.

[6] A. Gregori, C. Kounnas and P.M. Petropoulos, Nucl. Phys. B $\mathbf{5 5 3}(1999)$ 108.

[7] A. Sen and C. Vafa, iNucl. Phys. B $\mathbf{4 5 5}(1995)$ $165^{\prime}$

[8] A. Gregori, C. Kounnas and J. Rizos, 'íncl.' Phys. B $\mathbf{5 4 9}(1999) 16$

[9] I. Antoniadis, G. D'Appollonio, E. Dudas and A. Sagnotti, 'Nucl. Phys. B $\mathbf{5} \mathbf{5} \mathbf{3}(1999)$ 133'.

[10] A. Gregori and C. Kounnas, 'Nucl. Phys. B 560, $(1999)$ 135.

[11] E. Kiritsis, C. Kounnas, P.M. Petropoulos and J. Rizos, IPhys. Lett. B $385(1996) 87$. 